\title{
Suppression of HER-2 via siRNA interference promotes apoptosis and decreases metastatic potential of SKOV-3 human ovarian carcinoma cells
}

\author{
YAN-MING LU ${ }^{1}$, MENG-LI RONG ${ }^{1,2}$, CHAO SHANG ${ }^{3,4}$, NING WANG $^{1}$, \\ XIANG $\mathrm{LI}^{1}$, YAN-YAN ZHAO ${ }^{5}$ and SHU-LAN ZHANG ${ }^{1}$
}

\begin{abstract}
${ }^{1}$ Department of Gynaecology and Obstetrics, The Affiliated Shengjing Hospital, China Medical University, Shenyang; ${ }^{2}$ School of Health Sciences, Macao Polytechnic Institute, Macao; ${ }^{3}$ Department of Neurobiology, ${ }^{4}$ Institute of Pathology and Pathophysiology, China Medical University; ${ }^{5}$ Department of Clinical Genetics, Shengjing Hospital, China Medical University, Shenyang, P.R. China
\end{abstract}

Received September 28, 2012; Accepted December 3, 2012

DOI: 10.3892/or.2012.2214

\begin{abstract}
The aberrant expression of human epidermal growth factor receptor-2 (HER-2) has been detected in ovarian cancer. However, the role of HER-2 in the development of ovarian cancer has not been sufficiently elucidated. The objective of this study was to determine the role of HER-2 in the apoptosis and metastasis of SKOV-3 ovarian cancer cells. SKOV-3 cells were transfected with three double-stranded small interfering RNA (siRNA) molecules that target HER-2. Various sequences were synthesized by $\mathrm{T} 7$ transcription in vitro to select the most effective HER-2-silencing siRNA. SKOV-3 cells were examined for growth inhibition using the MTT proliferation assay and apoptosis was assessed using flow cytometry and TUNEL assay. The Matrigel basement memebrane matrix was used to assess invasion and chemotactic mobility, as a model of tumor cell metastasis. Western blot analysis was used to detect the expression of matrix metallopeptidase-9 (MMP-9), E-cadherin, $\mathrm{N}$-cadherin and vimentin. siRNA interference in HER-2 resulted in decreased cell proliferation and invasion and increased apoptosis. Western blot analysis demonstrated a marked increase in E-cadherin and MMP-9 and a reduction in N-cadherin and vimentin protein levels in the SKOV-3 cells. The suppression of HER-2 expression resulted in apoptosis and the inhibition of metastasis of SKOV-3 cells. Therefore, the overexpression of the HER-2 gene can enhance the metastatic potential of SKOV-3 cells by increasing the protein levels of MMP-9. Epithelial-mesenchymal transition may be involved in the HER-2 siRNA-induced invasion and migration of SKOV-3
\end{abstract}

Correspondence to: Dr Shu-Lan Zhang,Department of Gynaecology and Obstetrics, The Affiliated Shengjing Hospital, China Medical University, 36 Sanhao Street, Shenyang 110004, P.R. China E-mail: zs10909@sina.com

Key words: ovarian carcinoma, human epidermal growth factor receptor-2, apoptosis, migration, invasion, matrix metallopeptidase-9, epithelial-mesenchymal transition, E-cadherin cells. Taken together, these results suggest that HER-2 functions as an oncogene and may thus be an attractive therapeutic target in SKOV-3 ovarian cancer cells.

\section{Introduction}

Ovarian cancer has the highest mortality rate of all the gynecological cancers (1). The standard treatment is aggressive surgery followed by platinum and taxane-based chemotherapy. Due to the continuous improvement in surgical and diagnostic techniques, response rates have improved; however, the overall survival remains low (31\%) (2). Poor prognosis is mainly due to the late diagnosis of stage III and IV ovarian cancer and acquired chemoresistance from standard therapy. Therefore, a better understanding of the molecular mechanism of ovarian carcinogenesis and metastasis may provide insights for the development of novel therapeutic strategies.

The human epidermal growth factor receptor-2 (HER-2) gene is a member of the epidermal growth factor receptor (EGFR) family and encodes a $185-\mathrm{kDa}$ protein with tyrosine kinase activity $(3,4)$. The HER-2 protein can dimerize with other members of the EGFR family. Additionally, the overexpression of the HER-2 gene has been shown to enhance the kinase-mediated activation of downstream signaling pathways, such as mitogen-activated protein kinase (MAPK) and phosphatidylinositol-3 kinase (PI3K) (5). Experimental and clinical data have demonstrated that the overexpression or amplification of HER-2 correlate with tumorigenesis in a variety of tumors (6). The blockade of the HER-2 signaling pathway represents an opportunity for the development of novel and selective anticancer therapies $(7,8)$. Although previous studies have demonstrated that HER-2-blocking antibodies are somewhat effective, the clinical application of these treatments is controversial (9). HER-2 is one of the most widely characterized oncogenes linked with poor prognosis for ovarian cancer (10). Previous studies have shown that the overexpression of the HER-2 gene is found in approximately $30 \%$ of human ovarian cancers and correlates with a more advanced tumor stage and increased chemoresistance (11). 
RNA interference (RNAi) has become an excellent research tool for the silencing of target genes (12). Short interfering RNAs (siRNAs), 21-23 nucleotides (nt) in length, can silence the targeted gene by binding to complementary mRNA and interfering with gene expression (13). A previous study demonstrated that the transfection of synthetic 21-nt siRNA duplexes into mammalian cells efficiently inhibited endogenous gene expression in a sequence-specific manner (14). However, studies on the RNAi silencing of HER-2 expression and its role in ovarian cancer are limited.

In the present study, we designed siRNAs that targeted different regions within the open reading frame of HER-2 mRNA. We examined their ability to interfere with HER-2 gene expression, utilizing the HER-2-positive ovarian cancer cell line, SKOV-3. Specifically, we assessed HER-2 inhibition by examining cell proliferation, apoptosis, cell invasion and markers of epithelial-mesenchymal transition (EMT) in SKOV-3 cells.

\section{Materials and methods}

Cell lines and cell culture. The SKOV-3 ovarian cancer cell line was obtained from the China Center for Type Culture Collection (Wuhan, China). Cell culture was performed according to the manufacturer's instructions. The cells were routinely maintained in McCoy's 5A medium (Gibco-BRL, Invitrogen, Carlsbad, CA) supplemented with $10 \%$ fetal bovine serum (FBS) (Gibco-BRL) and $1 \%$ penicillin in a well-humidified incubator of $5 \% \mathrm{CO}_{2}$ at $37^{\circ} \mathrm{C}$.

siRNA preparation. HER-2 cDNA sequences were selected based on HER-2 target sites (obtained from GenBank) using siRNA design software from Invitrogen Life Technologies (Grand Island, NY). The siRNA target design tools from Ambion ${ }^{\circledR}$ were used to design the HER-2 siRNA, as well as non-specific siRNA sequences corresponding to the HER-2 gene, with $3^{\prime}$ overhanging UU dinucleotides. The sequences were designed for the regions of HER-2 mRNA that were the least homologous to the other HER-2 family members to ensure affinity. Briefly, the siRNAs were reconstituted in annealing buffer, as per the manufacturer's instructions, to obtain a $20-\mu \mathrm{M}$ solution. Three different regions within the HER-2 gene (NCBI accession no. M11730) were used in this study: siRNA-1 (positions 2215-2237), siRNA-2 (positions 2644-2666) and siRNA-3 (positions 2734-2756). siRNA-3S (scrambled) was constructed from random sequences of siRNA-3 and served as the control (depending on the experimental results). All siRNAs contained a 19-bp double-stranded (ds) sequence and symmetric 3 ' overhangs of two deoxythymidines. HER-2 siRNA-specific sequences were as follows: i) HER-2 siRNA-1 sense, 5'-GAUCC GGAAGUACACGAUGUU-3' and antisense, 5'-CAUCGUGU ACUUCCGGAUCUU-3'; ii) HER-2 siRNA-2 sense, 5'-GAGU CCCAACCAUGUCAAAUU-3' and antisense, 5'-UUUGACA UGGUUGGGACUCUU-3'; iii) HER-2 siRNA-3 sense, 5'-CUG GUGUAUGCAGAUUGCCUU-3' and antisense, 5'-GGCAA UCUGCAUACACCAGUU-3'; siRNA-3S sense, 5'-CUUGU AUGGGCAGAUUGCCUU-3' and antisense, 5'-GGCAAUC UGCCCAUACAAGUU-3'; and T7DNA template, 5'-CCTG TCTC-3'. All siRNAs were synthesized in vitro using T7 transcription.
siRNA transfection. Cells were plated in growth medium without antibiotics for $\sim 24 \mathrm{~h}$ prior to transfection. Transient transfection of siRNA was performed with Lipofectamine 2000 (Invitrogen) according to the manufacturer's instructions. The transfected cells were then cultured for $6 \mathrm{~h}$ and the culture medium was replaced with fresh medium supplemented with $10 \%$ FBS. The cells were harvested $24 \mathrm{~h}$ after transfection.

Reverse transcription-polymerase chain reaction (RT-PCR). Total RNA was extracted using TRIzol (Invitrogen) according to the manufacturer's instructions. For RT-PCR analysis, $5 \mu \mathrm{g}$ of total RNA was reverse-transcribed using RT-PCR kits (Promega, Madison, WI). PCR products were analyzed using standard agarose gel electrophoresis with ethidium bromide. The primer sequences used were as follows: forward primer, 5'-CCTGCTGAACTGGTGTATGCA-3' and reverse primer, 5'-TCAGAGTCAATCATCCAACATTTG-3'.

Western blot analysis. Total protein was extracted using radioimmunoprecipitation assay (RIPA) buffer. Protein $(50 \mu \mathrm{g})$ was loaded onto SDS-PAGE gels $(8-10 \%)$ and electrophoresed (200 V, 2 h), transferred onto PVDF membranes (BD Biosciences) and hybridized with primary antibodies, against HER-2, GAPDH, $\beta$-actin (1:1000, Zhongshan, Beijing, China), E-cadherin, vimentin, $\mathrm{N}$-cadherin and matrix metallopeptidase-9 (MMP-9) (1:1000, Santa Cruz Biotechnology, Santa Cruz, CA), followed by incubation with the appropriate goat anti-rabbit secondary antibodies conjugated with alkaline phosphatase (1:2000, Zhongshan). Antibody binding was detected using a BCIP/NBT kit (Zhongshan).

3-(4,5-Dimethylthiazol-2-yl)-2,5-diphenyltetrazolium bromide (MTT) assay. MTT assay (Sigma, St. Louis, MO) was performed to assess the silencing effect of HER-2 on SKOV-3 cell proliferation. Cells $(1,000 /$ well $)$ were plated in $96-$ well plates. The growth was monitored at various time-points $(24,48,72$ and $96 \mathrm{~h}$ ) and cell viability was measured using the MTT assay. Cell viability was expressed as optical density (OD). Absorbance was measured at $490 \mathrm{~nm}$ using a microplate reader (VersaMax, Molecular Devices, Sunnyvale, CA).

Annexin V apoptosis assay. The Annexin V-FITC-labeled Apoptosis Detection kit (Beijing Biosea Biotechnology) was used to assess apoptosis by flow cytometry, according to the manufacturer's instructions. At $48 \mathrm{~h}$ after transfection, a total of $5 \times 10^{5} \mathrm{SKOV}-3$ cells were transferred to a sterile tube, in which $5 \mu \mathrm{l}$ of Annexin $\mathrm{V}$ and $5 \mu \mathrm{l}$ of propidium iodide were added. The cells were then allowed to incubate at room temperature for $15 \mathrm{~min}$ and analyzed by flow cytometry (FACSCalibur, BD Biosciences, San Jose, CA).

Apoptosis in situ. The terminal deoxynucleotidyltransferasemediated dUTP-biotin nick end-labeling (TUNEL) assay was performed according to the manufacturer's instructions using the Promega DeadEnd ${ }^{\mathrm{TM}}$ fluorometric TUNEL system. Briefly, $1.5 \times 10^{6}$ SKOV-3 cells were plated using chamber slides and transfected with $2 \mu \mathrm{g}$ of siRNA-3. The cells were then washed with phosphate-buffered saline (PBS), fixed in $40 \mathrm{mg} / \mathrm{ml}$ paraformaldehyde solution for $25 \mathrm{~min}$ and then treated with $2 \mathrm{mg} / \mathrm{ml}$ Triton X-100 at $4^{\circ} \mathrm{C}$ for $5 \mathrm{~min}$ for permeabilization. 
A

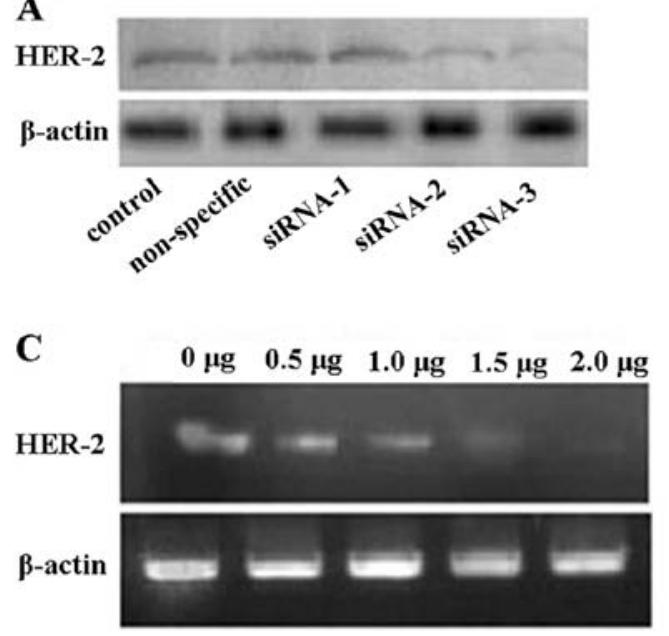

B

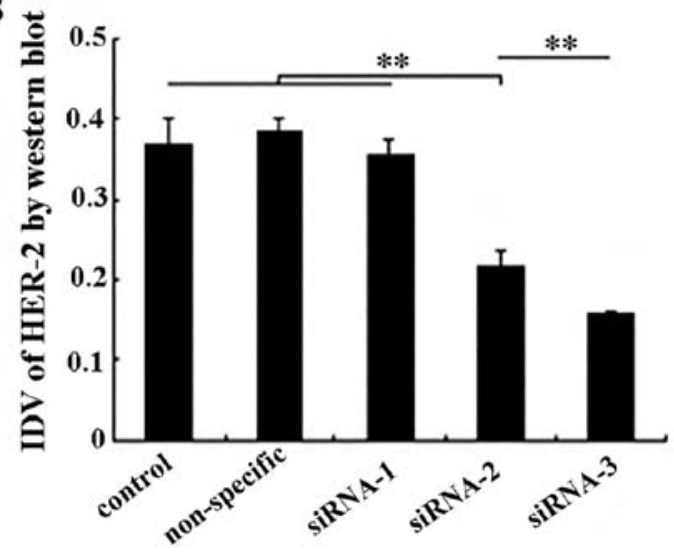

Figure 1. (A) Expression of the HER-2 protein by western blot analysis following transfection with three different HER-2 siRNAs. (B) Integrated density value (IDV) of HER-2 by western blot analysis. Strong interference effect of the HER-2 protein expression was achieved by transfection with siRNA-2 and siRNA-3 $\left({ }^{* *} \mathrm{P}<0.01\right)$. (C) Expression of HER-2 mRNA by RT-PCR following transfection with HER-2 siRNA-3 $(0.5,1.0,1.5,2.0 \mu \mathrm{g})$. The differences in the expression of HER-2 mRNA in each dose group were significant $(\mathrm{P}<0.01)$.

The cells were then washed and labeled with TUNEL reaction mixture at $37^{\circ} \mathrm{C}$ for $60 \mathrm{~min}$. After washing with saline-sodium citrate (SSC) buffer, cells were treated with DAPI. The chamber slides were evaluated using a fluorescence microscope (BD Biosciences) to assess for apoptotic cells indicated by strong green nuclear fluorescence. Apoptotic cells were determined by counting TUNEL-positive cells in five random fields for each sample. The apoptotic index (AI) was the number of apoptotic cells counted in ten high-powered (x400) fields $(\sim 1,000$ cells) that were randomly picked for quantification from the most intensely stained area of each section.

Invasion and migration assays. For the migration assay, $5 \times 10^{4}$ cells were suspended in serum-free McCoy's 5A medium and plated on chambers $(8-\mu \mathrm{m}$ pore size, Corning Costar, Cambridge, MA) that were not coated with Matrigel. For the invasion assay, the upper chamber was pre-coated with $5 \mathrm{mg} / \mathrm{ml}$ Matrigel (Sigma Aldrich) prior to the addition of $5 \times 10^{5}$ cells in serum-free McCcoy's 5A medium. For both assays, medium containing $10 \%$ FBS was added to the lower chamber as a chemoattractant. Following incubation for $18 \mathrm{~h}$ at $37^{\circ} \mathrm{C}$, non-invaded cells on the upper surface of the filter were wiped out with a cotton swab. The invading cells on the lower surface of the filter were fixed and stained with hematoxylin and eosin. The cell motility and invasiveness were determined by counting cells in five microscopic fields per well and the extent of invasion was expressed as the average number of cells per field.

Statistical analyses. All experiments represent an average of at least triplicate samples or as indicated. The data are presented as the means \pm SEM. All statistical analyses were carried out using Sigma Plot 13.0 software (SPSS, Chicago, IL). Differences between groups were assessed by one-way analysis of variance (ANOVA) (nonparametric statistics) and unpaired Student's t-tests. A value of $\mathrm{P}<0.05$ was considered to indicate a statistically significant difference.

\section{Results}

Screening and identification of the target site of HER-2 gene by $T 7$ in vitro transcription system. We examined the ability of specific HER-2 siRNAs to reduce the endogenous level of the HER-2 protein in SKOV-3 cells. We selected the SKOV-3 cell line since it is known to express high levels of endogenous HER-2 (15). We transfected SKOV-3 cells with three different HER-2-specific siRNAs. The western blot analysis results showed that the three HER-2-specific siRNAs had different levels of efficiency in downregulating the expression of the HER-2 protein (Fig. 1A). The greatest interference in HER-2 protein expression was achieved by transfection with siRNA-3 (57.7\%) and, to a lesser extent, with siRNA-2 (41.2\%). The control cells, or cells transfected with siRNA-1, exhibited no reduction in HER-2 protein levels (Fig. 1B). Thus, HER-2 siRNA-3 was chosen for the experimental studies. Furthermore, a non-specific siRNA-3 was designed to serve as the non-specific control group. Accordingly, the results showed that the non-specific siRNA-3 had no effect on HER-2 protein expression (Fig. 1B).

RT-PCR was used to compare the relative expression value of each siRNA-transfected group $(0.5,1.0,1.5$ and $2.0 \mu \mathrm{g})$, which demonstrated significant changes in HER-2 mRNA expression $(\mathrm{P}<0.01)$ (Fig. 1C). The expression of HER-2 mRNA correlated with the transfection amount of HER-2 siRNA-3. As shown in Fig. 1C, the expression of HER-2 mRNA was maximal with $2.0 \mu \mathrm{g}$ of transfected HER-2 siRNA-3. The siRNA interference was corroborated by western blot analysis of the HER-2 protein. Thus, $2.0 \mu \mathrm{g}$ of transfected siRNA-3 was used to investigate the characteristic changes of SKOV-3 cells in further experiments.

Silencing of HER-2 by siRNA-3 results in the inhibition of proliferation and the induction of apoptosis of SKOV-3 cells. The HER-2 gene is important for tumor development. Therefore, we investigated the role of HER-2 inhibition in SKOV-3 cell proliferation. As illustrated in Fig. 2A, HER-2 siRNA-3 exhibited a statistically significant growth-inhibitory effect 

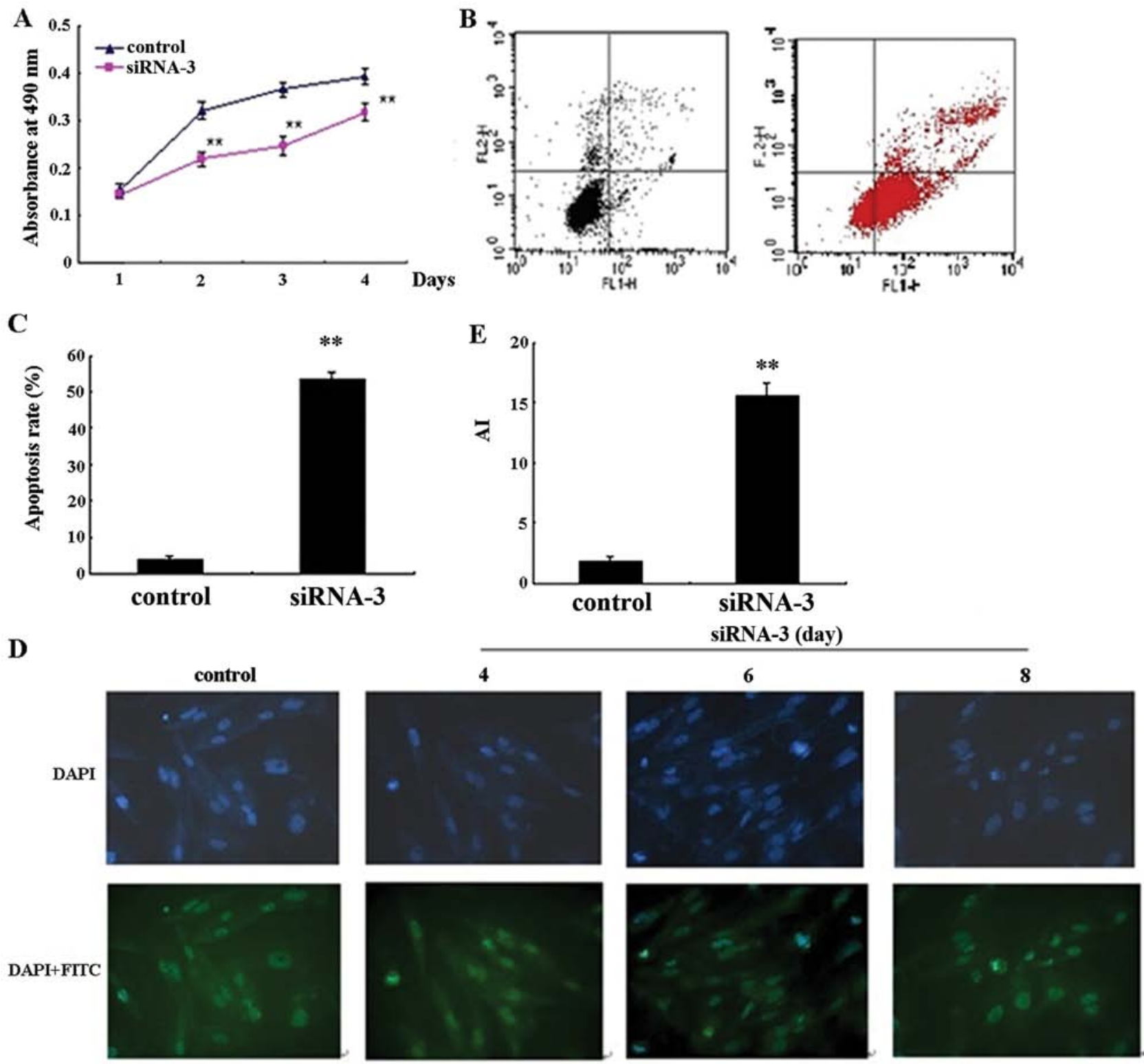

Figure 2. Effect of HER-2 siRNA-3 on SKOV-3 cell growth and apoptosis. (A) Growth-inhibitory effect of HER-2 siRNA-3 on SKOV-3 cells detected by MTT assay. Absorbance at $490 \mathrm{~nm}$ showed a significant decrease in the SKOV-3 cells compared to the control cells ( $\left.{ }^{* *} \mathrm{P}<0.01\right)$. (B) Apoptosis was analyzed by flow cytometry. (C) The percentage of apoptotic cells is shown indicating the highest percentage of apoptotic cells among the HER-2 siRNA3-transfected cells compared to the control cells, which is graphically shown in (C). (D) Cell morphology of siRNA3-transfected cells by TUNEL assay (on the 4th, 6th and 8th day). Cytoplasmic condensation, mottled appearance of the nucleus and diverse morphology, showing the formation of apoptotic bodies in the specific interference group, which is graphically shown in (E). AI, apoptotic index.

on SKOV-3 cells when evaluated by MTT assay. Additionally, HER-2 siRNA-3 resulted in a significant decrease in cell growth compared to the control cells at various time-points $(24,72$ and $96 \mathrm{~h})(\mathrm{P}<0.01)$. To determine whether apoptosis could be induced by HER-2 siRNA-3, we assessed apoptosis by Annexin V staining and flow cytometry. As illustrated in Fig. 2B, the percentage of apoptotic SKOV-3 cells increased following transfection with HER-2 siRNA-3 and significantly increased compared to the control cells $(\mathrm{P}<0.01$, Fig. $2 \mathrm{C})$.

To further evaluate apoptosis and morphological changes caused by HER-2 siRNA-3 transfection, we analyzed SKOV-3 cells using TUNEL assay at different time-points $(96,144$, and $192 \mathrm{~h})$. The number of apoptotic cells after siRNA-3 transfection was the highest at $192 \mathrm{~h}$ compared to the control group
$(\mathrm{P}<0.01)$ (Fig. 2D). Collectively, the data from Annexin $\mathrm{V}$ and TUNEL analysis demonstrate that siRNA-3 targeting of HER-2 induced apoptosis in SKOV-3 cells.

Effects of HER-2 siRNA-3 on SKOV-3 cell migration and invasion. Enhanced cell migration is characteristic of cancer cells during EMT. Invasion and migration assays were performed to assess cell invasion and the migration ability of HER-2 siRNA-transfected SKOV-3 cells.

As shown in Fig. 3A, HER-2 siRNA-3 induced a significant decrease in the number of cells that invaded through a reconstituted basement membrane. The invasion and chemotactic capacity were decreased in HER-2 siRNA-transfected SKOV-3 cells $(\mathrm{P}<0.01)$ (Fig. 3B and $\mathrm{C})$. We also examined the effect 
$\mathbf{A}$

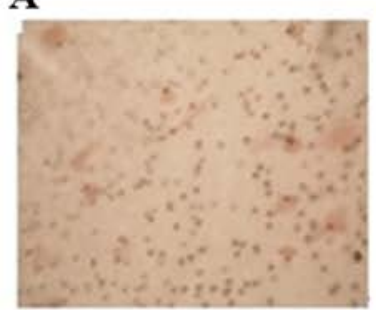

control

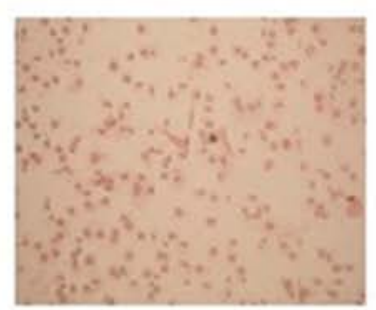

siRNA-3
B

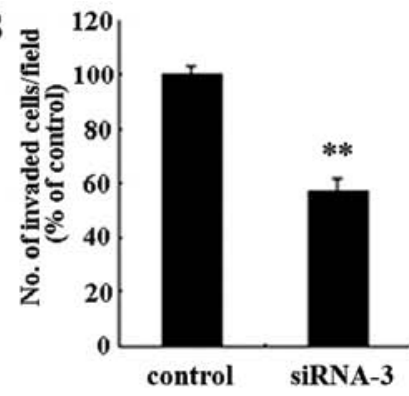

C

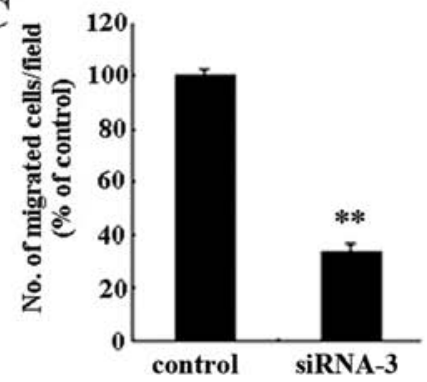

$\mathbf{E}$

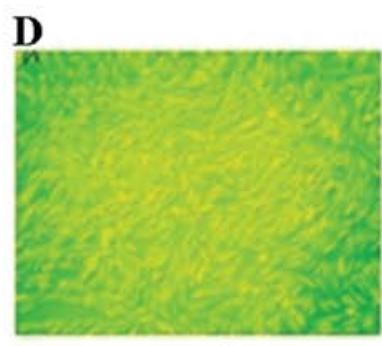

control

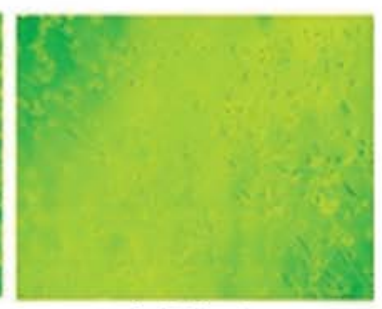

siRNA-3

Figure 3. Effects of HER-2 siRNA3 on SKOV-3 cell migration and invasion. (A) Cells were evaluated using the Matrigel invasion assay. HER-2 siRNA3 induced a significant decrease in the number of cells that invaded through a reconstituted basement membrane. (B) The number of invading cells per field was counted (x200) in an arbitrary visual field. (C) The number of migrated cells per field was counted (x200) in an arbitrary visual field. The invasion and chemotactic mobile capacity of SKOV-3 cells were decreased after HER-2 siRNA transfection ( $\left.{ }^{* *} \mathrm{P}<0.01\right)$. (D) Morphological alteration of SKOV-3 cells after HER-2 siRNA transfection was observed under an inverted microscope. The shape of the SKOV-3 cells changed from an elongated spindle-like to a cuboidal shape, a morphology typical of epithelial-like cells. (E) Cell lysates were analyzed for the expression of E-cadherin, N-cadherin, vimentin and MMP-9 by western blot analysis.

of HER-2 siRNA-3 on the expression of MMP-9 by western blot analysis, which showed a marked decrease (Fig. 3E). Based on these results, the silencing of HER-2 may inhibit the cell migration and invasion of SKOV-3 cells via the downregulation of MMP-9.

Effects of HER-2 siRNA-3 on expression of EMT markers in SKOV-3 cells. Silencing HER-2 expression can induce morphological changes in SKOV-3 cells. As shown in Fig. 3D the shape of the cells changed from an elongated spindle-like shape to a cuboidal one, a morphology typical of epithelial-like cells. To determine whether HER-2 siRNA affected specific molecular changes of EMT in SKOV-3 cells, we assessed the protein levels of E-cadherin, vimentin and $\mathrm{N}$-cadherin. As illustrated in Fig. 3E, E-cadherin levels were increased, while vimentin and $\mathrm{N}$-cadherin levels were decreased in the transfected cells. These results indicate that the HER-2 siRNA-3-induced morphological changes may involve EMT in SKOV-3 cells (Fig. 3E).

\section{Discussion}

The amplification and overexpression of the HER-2 gene have been associated with many human cancers, such as breast and ovarian cancers, as well as with the poor prognosis of ovarian carcinoma $(7,8)$. In this study, we used the $\mathrm{T} 7$ in vitro transcription system to silence the HER-2 gene with siRNAs in SKOV-3 cells. Three specific pairs of HER-2-targeted sequences were devised using the coding region of HER-2 mRNA. We found that all three siRNAs were efficient in downregulating the expression of HER-2. However, HER-2 siRNA-3 was the most effective suppressor of HER-2 protein expression.

HER-2 is involved in numerous cellular functions, including apoptosis and cell cycle progression $(5,6)$. In the present study, the inhibition of HER-2 resulted in a significant decrease in the growth of SKOV-3 cells. Apoptosis has been reported to play an important role during the malignant transformation of normal cells $(16,17)$. These findings, in conjunction with ours, suggest that the dysregulation of HER-2 disrupts the balance between proliferation and apoptosis and may represent an important pathogenic step in the development of ovarian cancer. These changes were observed when the oncogene was neutralized in HER-2-overexpressing tumors or antisense oligonucleotides $(8,18-20)$.

Metastasis is an important characteristic of malignant tumors. An essential part of invasion and metastasis is the degradation of the basement membrane by members of the MMP family (21). MMPs belong to a family of zinc-dependent proteinases that have the ability to degrade extracellular matrix components. Accumulating evidence has suggested a strong correlation exists between high levels of MMPs and malignant cancer invasiveness (22). Recent clinical studies have indicated that there is an association between HER-2 expression and MMP-9 production in cancer patients $(23,24)$. Kim et al, as well as others, demonstrated that the overexpression of the HER-2 gene induced the invasion of human breast epithelial cells via MMP-9 $(24,25)$. In this study, we showed that the suppression of HER-2 by siRNA inhibited the migration and invasiveness of ovarian carcinoma cells. Decreased MMP-9 protein expression was also detected by western blot analysis. 
The results of the present study suggest that overexpression of the HER-2 gene may promote the metastatic potential of SKOV-3 cells expressing MMP-9.

EMT, which has been recognized as a crucial feature of embryogenesis, has recently been shown to be closely related with carcinoma cell migration and invasion (26). Previous studies have focused on the mechanisms and regulation of HER-2-driven EMT of mammary tumor cells $(27,28)$. In addition, recent studies have shown that EGFR causes EMT in cervical, ovarian and liver cancers (29-31). During EMT, the polarized and basal membrane-anchored epithelial cells undergo a number of biochemical changes to acquire a mesenchymal, fibroblastoid phenotype. In our study, silencing HER-2 expression induced morphological changes in SKOV-3 cells, whose shape changed from an elongated spindle-like to a cuboidal shape, a morphology typical of epithelial-like cells. The strongest evidence for the significance of EMT relates to the cell adhesion molecule, E-cadherin (epithelial cadherin). Abolishing E-cadherin function in vitro confers invasive properties to non-invasive cells and, conversely, the introduction of E-cadherin into invasive epithelial cell lines abrogates their invasive potential. In this regard, E-cadherin is considered a broadly acting suppressor of invasion and metastasis and its functional inactivation represents a critical step in the acquisition of this capability (16). Not surprisingly, loss of E-cadherin expression is a defining characteristic of EMT (32). N-cadherin (neural cadherin), another adhesion molecule, is associated with a heightened invasive potential in cancer. In addition, vimentin, a mesenchymal marker, is significantly associated with the expression of $\mathrm{N}$-cadherin (33). In this study, E-cadherin levels were increased and the expression of vimentin and $\mathrm{N}$-cadherin was decreased following transfection with HER-2 siRNA-3, indicating that the HER-2 siRNA-3-induced morphological changes may involve EMT in SKOV-3 cells. Considering the emerging importance of EMT in invasive cell behavior, the detailed mechanisms for the role of EMT in HER-2-driven malignancy in SKOV-3 cells require further elucidation.

The HER-2 gene has been identified to play important roles in chemoresistance in a number of tumors. In a previous study conducted by our laboratory, we investigated the inhibition of HER-2 by siRNA in SKOV-3 cells and its role in potentiating the efficacy of cisplatin. Our findings demonstrated that cisplatin inhibited cellular proliferation in a dose-dependent manner (34). These results are consistent with growing evidence suggesting that the activation of the HER-2 signal transduction pathway may induce chemoresistance in SKOV-3 cells (35).

In conclusion, the siRNA-mediated interference of HER-2 expression inhibited the proliferation, induced apoptosis and inhibited invasive and migratory phenotypes of SKOV-3 cells. Given that HER-2 is one of the most important oncogenes in human ovarian cancer and an attractive therapeutic target, our findings provide a molecular basis for further evaluation of the role of HER-2 in ovarian cancer progression.

\section{Acknowledgements}

This study was supported by the National Natural Science Foundation of China (81001163), the Liaoning Education
Department for Scientific and Technological Research Project funds (2009A781) and the Scientific Research Foundation of Shengjing Hospital of China Medical University.

\section{References}

1. Jemal A, Siegel R, Xu J and Ward E: Cancer statistics, 2010. CA Cancer J Clin 60: 277-300, 2010.

2. Jemal A, Siegel R, Ward E, Hao Y, Xu J and Thun MJ: Cancer statistics, 2009. CA Cancer J Clin 59: 225-249, 2009.

3. Olayioye MA, Neve RM, Lane HA and Hynes NE: The ErbB signaling network: receptor heterodimerization in development and cancer. EMBO J 19: 3159-3167, 2000.

4. Baselga J and Arteaga CL: Critical update and emerging trends in epidermal growth factor receptor targeting in cancer. J Clin Oncol 23: 2445-2459, 2005.

5. Yarden Y and Sliwkowski MX: Untangling the ErbB signalling network. Nat Rev Mol Cell Biol 2: 127-137, 2001.

6. Hynes NE and Lane HA: ERBB receptors and cancer: the complexity of targeted inhibitors. Nat Rev Cancer 5: 341-354, 2005.

7. Duchnowska R, Biernat W, Szostakiewicz B, et al: Correlation between quantitative HER-2 protein expression and risk for brain metastases in HER $-2^{+}$advanced breast cancer patients receiving trastuzumab-containing therapy. Oncologist 17: 26-35, 2012.

8. Howe LR and Brown PH: Targeting the HER/EGFR/ErbB family to prevent breast cancer. Cancer Prev Res 4: 1149-1157, 2011.

9. Modjtahedi $\mathrm{H}$ and Essapen S: Epidermal growth factor receptor inhibitors in cancer treatment: advances, challenges and opportunities. Anticancer Drugs 20: 851-855, 2009.

10. Serrano-Olvera A, Dueñas-González A, Gallardo-Rincón D, Candelaria $M$ and De la Garza-Salazar J: Prognostic, predictive and therapeutic implications of HER 2 in invasive epithelial ovarian cancer. Cancer Treat Rev 32: 180-190, 2006.

11. Rubin SC, Finstad CL, Wong GY, Almadrones L, Plante M and Lloyd KO: Prognostic significance of HER-2/neu expression in advanced epithelial ovarian cancer: a multivariate analysis. Am J Obstet Gynecol 168: 162-169, 1993.

12. Fire A, Xu S, Montgomery MK, Kostas SA, Driver SE and Mello CC: Potent and specific genetic interference by stranded RNA in Caenorhabditis elegans. Nature 391: 806-811, 1998.

13. Szweykowska-Kulińska Z, Jarmołowski A and Figlerowicz M: RNA interference and its role in the regulation of eucaryotic gene expression. Acta Biochim Pol 50: 217-229, 2003.

14. Elbashir SM, Harborth J, Lendeckel W, Yalcin A, Weber K and Tuschl T: Duplexes of 21-nucleotide RNAs mediate RNA interference in cultured mammalian cells. Nature 411: 494-498, 2001.

15. Ueno NT, Bartholomeusz C, Herrmann JL, et al: E1A-mediated paclitaxel sensitization in HER-2/neu-overexpressing ovarian cancer SKOV3.ip1 through apoptosis involving the caspase-3 pathway. Clin Cancer Res 6: 250-259, 2000.

16. Hanahan D and Weinberg RA: Hallmarks of cancer: the next generation. Cell 144: 646-674, 2011.

17. Thompson CB: Apoptosis in the pathogenesis and treatment of disease. Science 267: 1456-1462, 1995.

18. Chuang TC, Hsu SC, Cheng YT, Shao WS, Wu K, Fang GS, Ou CC and Wang V: Magnolol down-regulates HER2 gene expression, leading to inhibition of HER2-mediated metastatic potential in ovarian cancer cells. Cancer Lett 311: 11-19, 2011.

19. Yamashita-Kashima Y, Iijima S, Yorozu K, Furugaki K, Kurasawa M, Ohta M and Fujimoto-Ouchi K: Pertuzumab in combination with trastuzumab shows significantly enhanced antitumor activity in HER2-positive human gastric cancer xenograft models. Clin Cancer Res 17: 5060-5070, 2011.

20. Tanizaki J, Okamoto I, Fumita S, Okamoto W, Nishio K and Nakagawa K: Roles of BIM induction and survivin downregulation in lapatinib-induced apoptosis in breast cancer cells with HER2 amplification. Oncogene 30: 4097-4106, 2011.

21. Decock J, Thirkettle S, Wagstaff L and Edwards DR: Matrix metalloproteinases: protective roles in cancer. J Cell Mol Med 15: 1254-1265, 2011.

22. Hua H, Li M, Luo T, Yin Y and Jiang Y: Matrix metalloproteinases in tumorigenesis: an evolving paradigm. Cell Mol Life Sci 68: 3853-3868, 2011.

23. Luukkaa H, Klemi P, Leivo I, et al: Expression of matrix metalloproteinase-1, -7, -9, -13, Ki-67, and HER-2 in epithelialmyoepithelial salivary gland cancer. Head Neck 32: 1019-1027, 2010. 
24. Kim IY, Yong HY, Kang KW and Moon A: Overexpression of ErbB2 induces invasion of MCF10A human breast epithelial cells via MMP-9. Cancer Lett 275: 227-233, 2009.

25. Tan M, Yao J and Yu D: Overexpression of the c-erbB-2 gene enhanced intrinsic metastasis potential in human breast cancer cells without increasing their transformation abilities. Cancer Res 57: 1199-1205, 1997.

26. Chua KN, Poon KL, Lim J, Sim WJ, Huang RY and Thiery JP Target cell movement in tumor and cardiovascular diseases based on the epithelial-mesenchymal transition concept. Adv Drug Deliv Rev 63: 558-567, 2011.

27. Sethi S, Sarkar FH, Ahmed Q, et al: Molecular markers of epithelial-to-mesenchymal transition are associated with tumor aggressiveness in breast carcinoma. Transl Oncol 4: 222-226, 2011.

28. Jenndahl LE, Isakson P and Baeckström D: c-erbB2-induced epithelial-mesenchymal transition in mammary epithelial cells is suppressed by cell-cell contact and initiated prior to E-cadherin downregulation. Int J Oncol 27: 439-448, 2005.

29. Ricono JM, Huang M, Barnes LA, et al: Specific cross-talk between epidermal growth factor receptor and integrin alphavbeta5 promotes carcinoma cell invasion and metastasis. Cancer Res 69: 1383-1391, 2009.
30. Ahmed N, Maines-Bandiera S, Quinn MA, Unger WG, Dedhar S and Auersperg N: Molecular pathways regulating EGF-induced epithelio-mesenchymal transition in human ovarian surface epithelium. Am J Physiol Cell Physiol 290: C1532-C1542, 2006.

31. Fuchs BC, Fujii T, Dorfman JD, Goodwin JM, Zhu AX, Lanuti $\mathrm{M}$ and Tanabe KK: Epithelial-to-mesenchymal transition and integrin-linked kinase mediate sensitivity to epidermal growth factor receptor inhibition in human hepatoma cells. Cancer Res 68: 2391-2399, 2008.

32. Thiery JP: Epithelial-mesenchymal transitions in tumour progression. Nat Rev Cancer 2: 442-454, 2002.

33. Vergara D, Merlot B, Lucot JP, Collinet P, Vinatier D, Fournier I and Salzet M: Epithelial-mesenchymal transition in ovarian cancer. Cancer Lett 291: 59-66, 2010.

34. Lu YM, Zhang SL, Meng LR and Zhao YY: Influence of human epidermal growth factor receptor-2 siRNA on chemosensitivity to cisplatin of human ovarian carcinoma cells: an in vitro experiment. Zhonghua Yi Xue Za Zhi 88: 909-913, 2008 (In Chinese).

35. Abuharbeid S, Apel J, Zugmaier G, et al: Inhibition of HER-2 by three independent targeting strategies increases paclitaxel resistance of SKOV-3 ovarian carcinoma cells. Naunyn Schmiedebergs Arch Pharmacol 371: 141-151, 2005. 